\title{
Fuzzy phase partition and hybrid modeling based quality prediction and process monitoring methods for multiphase batch processes
}

\author{
Lijia Luo, ${ }^{\dagger+}$ Shiyi Bao, ${ }^{* \dagger}$ Jianfeng Mao, ${ }^{\dagger}$ Di Tang, ${ }^{\dagger}$ and Zengliang Gao ${ }^{\dagger}$ \\ †Institute of Process Equipment and Control Engineering, Engineering Research Center of Process \\ Equipment and Remanufacturing, Ministry of Education, Zhejiang University of Technology, Hangzhou,
} Zhejiang 310014, China

${ }^{\sharp}$ Department of Chemical and Biomolecular Engineering, University of Delaware, Newark, Delaware 19716, United States

\section{Supporting Information}

Conclusions in the columns named "Computation burden", "Partition accuracy" and "Practicability and universality" in Table 1 are explained as follows:

Computation burden: The MP algorithm firstly builds a complete PCA or PLS model for the entire process, and then divides it into several sub-models to get the best prediction performance. Each sub-model corresponds to an operation phase. However, searching appropriate model division points is very time-consuming, because it searches over all sampling instants more than once to find the best division points. Beside, each sub-model is further optimized by increasing or decreasing the number of principal components. Therefore, the MP algorithm has heavy computation burden.

Sub-PCA/PLS and STMPCA firstly build a time-slice PCA/PLS model for each time-slice data matrix, and then classify loading matrices of time-slice PCA/PLS models using the K-means (KM) clustering 
algorithm. Finally, clustering results of the KM algorithm are post-processed to generate the final phase partition results. Therefore, Sub-PCA/PLS and STMPCA have medium computation burden.

The GMM-based phase partition method carries out phase partition by building a Gaussian mixture model. Each Gaussian component represents an operation phase. Samples are classified into different operation phases according to the posterior probability. The computation burden of building a Gaussian mixture model is approximately equal to that of performing the fuzzy c-mean clustering (FCM) algorithm. However, the GMM-based phase partition method also needs a postprocessing procedure to generate the final phase partition results. Therefore, it has medium computation burden.

The SCFCM-based phase partition method carries out phase partition by performing the SCFCM clustering algorithm on the trajectory data of process variables. The computation burden of the SCFCM algorithm is approximately equal to or less than that of the FCM algorithm. Therefore, the SCFCM-based phase partition method has slight computation burden.

Partition accuracy: MP, sub-PCA/PLS, STMPCA and GMM are correlation-based methods. They carry out phase partition by detecting changes in latent variable correlations. These correlation-based methods use some modeling approaches, such as PCA, PLS or GMM, to reveal latent variable correlations in process data. However, the revealed variable correlations cannot accurately describe the actual process mechanism, probably decreasing the phase partition accuracy. Besides, sub-PCA/PLS, STMPCA and GMM cannot cope with the sequentiality of data. Because of this drawback, their raw phase partition results may contain some exceptional time instants that destroy the continuity of operation phases. To generate consecutive operation phases, those exceptional time instants are assigned into appropriate phases 
by post-processing. However, this post-processing procedure may reduce the phase partition accuracy.

The SCFCM-based phase partition method is a trajectory-based method, which carries out phase partition by detecting changes in variable trajectories. The SCFCM-based method has higher phase partition accuracy, benefiting from the quick and accurate responses of phase-sensitive variables to changes in operation phases. In addition, the SCFCM-based method can cope with the sequentiality of data, directly generating consecutive operation phases with no need for any post-processing procedure. This also makes the SCFCM-based method have higher phase partition accuracy.

Practicability and universality: As mentioned above, the correlation-based phase partition methods use some modeling approaches to reveal latent variable correlations in process data. However, this modeling procedure not only increases the computational burden, but also leads to three drawbacks: (i) using different modeling approaches may generate different phase partition results for the same batch process; (ii) one modeling approach is only suitable for some specific batch processes; (iii) additional model parameters need to be determined. These three drawbacks hinder the implementation and popularization of correlation-based methods. Therefore, the correlation-based phase partition methods have low practicability and universality.

In the SCFCM-based method, process data are directly used for phase partition and no modeling approach is required. Therefore, the SCFCM-based method overcomes above three drawbacks in correlation-based methods, and it is much easier to implement and popularize. It thus has high practicability and universality. 